\title{
Influencia del tamaño del material híbrido en las características de los oxicarburos de silicio obtenidos
}

\author{
M. A. MAZO' ${ }^{1}$, C. PALENCIA 1 , A. NISTAL ${ }^{1}$, F. RUBIO' ${ }^{1}$, J. RUBIO ${ }^{1}$, J.L. OTEO ${ }^{1}$ \\ 'Instituto de Cerámica y Vidrio. Consejo Superior de Investigaciones Científicas. C/ Kelsen 5, 28049 Madrid, España.
}

\begin{abstract}
En este trabajo se ha investigado cómo influye el tamaño de los materiales híbridos del sistema TEOS/PDMS en las características de los vidrios de oxicarburo de silicio obtenidos. Los híbridos en piezas monolíticas (100 $\mathrm{mm} \times 50 \mathrm{~mm} \times 5 \mathrm{~mm}$ ) fueron triturados $(1 \mathrm{~mm} \times 1 \mathrm{~mm} \times 1 \mathrm{~mm})$ o molidos en mortero de ágata o atrición hasta $16 \mu \mathrm{m}$ o $3 \mu \mathrm{m}$, respectivamente. Posteriormente, se pirolizaron a $1100{ }^{\circ} \mathrm{C}$, obteniéndose los correspondientes vidrios de oxicarburo de silicio (SiOC). En estos SiOC se han encontrado diferencias en función del tamaño del material híbrido, diferencias que han sido estudiadas desde un punto de vista estructural y microestructural. La muestra monolítica presentó un mayor \% C en red (34 \%) y, a medida que se muele en mortero de ágata, disminuye hasta el $26 \%$. Sin embargo, para la muestra de atrición se obtiene valores similares a la monolítica (32\%). Esto concuerda con la presencia de la banda de FT-IR situada a $880 \mathrm{~cm}^{-1}$ atribuida a enlaces Si-C debidos a una mayor incorporación de carbono en la red. La fase de carbono libre en la muestra monolítica posee un dominio de 3.44 $\mathrm{nm}$ que aumenta hasta $3.66 \mathrm{~nm}$ en la de atrición. Este aumento del dominio corresponde al aumento de la grafitización. La morfología del SiOC monolítico corresponde a agregado de partículas esféricas con poros de $12 \mu \mathrm{m}$. Estos poros desaparecen en el SiOC de atrición, el cual está formado por partículas irregulares de $3 \mu \mathrm{m}$.
\end{abstract}

Palabras clave: Hibrido, tamaño, pirolisis, oxicarburo de silicio, porosidad

Influence of the hybrid material size in silicon oxycarbide materials characteristics.

In this work has been investigated the influence of the TEOS/PDMS hybrid size during the pyrolysis process to obtain the related silicon oxycarbide glasses. Hybrids monolithic pieces $(100 \mathrm{~mm} \times 50 \mathrm{~mm} \times 5 \mathrm{~mm})$, were crushed $(1 \mathrm{~mm} \times 1 \mathrm{~mm}$ $x 1 \mathrm{~mm}$ ) and grounded in agate or attrition to 16 and $3 \mu \mathrm{m}$, respectively. Then, they were pyrolyzed at $1100{ }^{\circ} \mathrm{C}$ to obtain silicon oxycarbide glasses ( $\mathrm{SiOC}$ ). These materials present structural and microstructural differences. The monolithic sample showed the highest \% C into the silica network (34\%), and after grounding in agate mortar decreased to $26 \%$. However the attrition milled sample reached \%C values similar to the monolithic (32\%). This agrees with FT-IR band at $880 \mathrm{~cm}^{-1}$ assigned to $\mathrm{Si}-\mathrm{C}$ bonds formation and related to higher carbon content into silica network. The carbon free phase displays a domain size of $3.44 \mathrm{~nm}$ which increased to $3.66 \mathrm{~nm}$ for the attrition milled. This result is due to a higher graphitization degree. The SiOC monolithic sample morphology displays spherical interconnected particles with pores of $12 \mu \mathrm{m}$. This morphology disappeared for the SiOC attrition milled sample, formed by irregular particles of $3 \mu \mathrm{m}$.

Key words: Hybrid, size, pyrolysis, silicon oxycarbide, porosity

\section{INTRODUCCIÓN}

Los oxicarburos de silicio (SiOC) son una nueva familia de materiales vítreos con enlaces Si-O-Si donde los átomos de oxígeno han sido parcialmente reemplazados por átomos de carbono (1). Esta incorporación de carbono en la red vítrea supone un fortalecimiento de dicha red, lo que produce tanto una mejora de sus propiedades con respecto a la sílice vítrea como la aparición de nuevas propiedades que anteriormente no se habían apreciado en este tipo de materiales (2). Estos materiales presentan unas buenas propiedades mecánicas $(3,4,5,6)$ térmicas (resistencia a la fluencia a elevadas temperaturas) $(7,8)$, resistencia frente a la oxidación $(9,10,11,12)$ estabilidad química a elevadas temperatura o frente al ataque de ácidos y bases $(13,14)$. Recientemente, se han encontrado otras características tales como que presentan un comportamiento viscoelástico a elevada temperatura (15), así como propiedades piezoeléctricas (16).
Este tipo de materiales se obtienen mediante pirolisis en atmósfera inerte a temperaturas relativamente bajas ( $1000{ }^{\circ} \mathrm{C}$ ) tanto de híbridos orgánico-inorgánicos, obtenidos mediante el proceso sol-gel, como de polímeros precerámicos, obtenidos mediante la ruta polimérica $(1,17)$. Los precursores, además de presentar enlaces Si-O, también tienen enlaces $\mathrm{Si}-\mathrm{C}_{\text {orgánico }}$ los cuales, durante el proceso de pirolisis, se transforman en enlaces Si-C-Si formadores de la estructura del vidrio de oxicarburo de silicio. La matriz del SiOC vidrio de oxicarburo está compuesta por una distribución aleatoria de enlaces Si-O, Si-C $(18,19)$, formando diferentes unidades estructurales $\left(\left[\mathrm{SiO}_{4}\right],\left[\mathrm{SiCO}_{3}\right],\left[\mathrm{SiC}_{2} \mathrm{O}_{2}\right],\left[\mathrm{SiC}_{3} \mathrm{O}\right]\right.$ y $\left.\left[\mathrm{SiC}_{4}\right]\right)$, donde no se encuentran presentes enlaces $\mathrm{Si}-\mathrm{Si}, \mathrm{C}-\mathrm{O}$ o $\mathrm{C}-\mathrm{C}$. Como consecuencia de esta manera de obtención se produce la transformación orgánico-inorgánica del material formándose el vidrio de oxicarburo, pero a su vez también se forma lo que 
se conoce como carbono libre, que es carbono tipo grafito, que está embebido dentro de la matriz vítrea (20).

Este tipo de materiales puede ser descrito mediante la siguiente fórmula estequiométrica, la cual incluye la fase oxicarburo y la fase de carbono libre adicional (21) (Fórmula I).

$$
\operatorname{SiC}_{\mathrm{x}} \mathrm{O}_{2(1-\mathrm{x})}+\mathrm{yC}_{\text {libre }} \quad \text { Fórmula I }
$$

Los vidrios de oxicarburo son amorfos, según se determina mediante difracción de rayos-X. Sin embargo, para temperaturas superiores a $1200{ }^{\circ} \mathrm{C}$ (en función de su composición) se produce una segregación de la fase metaestable de SiOC hacia la formación de sílice y carburo de silicio. Para temperaturas superiores, del orden de los $1500^{\circ} \mathrm{C}$, la silice reacciona con el carbono libre formando más carburo de silicio y especies volátiles $(1,22)$.

El proceso de pirolisis, es decir la transformación desde el material híbrido (o polímero) hasta el vidrio de oxicarburo de silicio, está influido por numerosos factores que determinan el material final, así como sus posibles aplicaciones. Los factores más importantes son: la naturaleza del precursor orgánico-inorgánico (naturaleza de los grupos orgánicos que lo forman, relación $(\mathrm{O} / \mathrm{Si})$, presencia de enlaces $\mathrm{Si}-\mathrm{H}$ o alcóxidos metálicos, microestructura) y las condiciones del tratamiento (tipo de atmósfera, temperatura, velocidad o el tiempo de permanencia) (23).

En la literatura pueden encontrarse trabajos donde se ha estudiado la formación de estos materiales con espesores normalmente pequeños, a la vez que se han llevado a cabo estudios de cómo influye la temperatura o el tipo de atmósfera en la formación de recubrimientos y films de carburo de silicio y oxicarburo de silicio (24). Otros estudios han analizado membranas de vidrios de oxicarburo de titanio con un espesor de $200 \mu \mathrm{m}$, y se ha visto cómo influye la porosidad en la difusividad en función del contenido en titanio (25). Por otro lado, Soraru et al. (7) establecieron que el tamaño de partícula influía en la estabilidad térmica de vidrios de oxicarburo una vez formados, observando como para muestras molidas por debajo de $100 \mu \mathrm{m}$ disminuía la estabilidad térmica a temperaturas superiores a $1400{ }^{\circ} \mathrm{C}$. Finalmente, Parmentier et. al (26). Observaron que mediante modificaciones durante el proceso sol-gel se obtenían híbridos con diferente microestructura los cuales daban lugar a vidrios de oxicarburo de silicio que presentaban diferentes comportamientos tanto frente a la oxidación como a elevadas temperaturas. Dichos autores observan que una propiedad clave es la porosidad, pues ésta promueve la degradación del material, mientras que si el material es denso y de composición similar al poroso, aquél es más resistente.

En los últimos años ha habido un gran desarrollo de materiales de vidrio de oxicarburo en diferentes tamaños y formas en función de cual sea su aplicación. Estos pueden desarrollarse como materiales estructurales de baja densidad, monolitos, matrices de materiales compuestos, espumas, fibras, membranas para separación de gases, partículas como adsorbentes para catalizadores, precursores de carburo de silicio de alta pureza, etcétera. $(1,7,17)$ Esta gran diversidad de tamaños y formas en función del tipo de aplicación final que vaya a tener el material, hace que sea necesario llevar a cabo un estudio de cómo influye el tamaño del material precursor en las características del material final obtenido. Este trabajo se ha centrado en ver cómo influye el tamaño del material híbrido orgánico-inorgánico de partida, ya sea monolito, trozos o polvo (de mayor o menor tamaño), en las características de los vidrios de oxicarburo de silicio obtenidos.

\section{PROCEDIMIENTO EXPERIMENTAL}

\subsection{Preparación de los vidrios de oxicarburo}

Los materiales híbridos orgánico-inorgánicos se obtuvieron a partir del proceso sol-gel empleando tetraetilortosilicato (TEOS; Merck) y polidimetilsiloxano (PDMS; ABCR; peso molecular $1750 \mathrm{gmol}^{-1}$ ). Se utilizó isopropanol (i-PrOH; Merck) como disolvente, $\mathrm{H}_{2} \mathrm{O}$ como agente hidrolizante y ácido clorhídrico ( $\mathrm{HCl}$; Merck; pureza del $32 \%$ ) como catalizador. La relación en peso TEOS/PDMS fue 60/40 y las relaciones molares TEOS/i-PrOH/ $\mathrm{H}_{2} \mathrm{O} / \mathrm{HCl}$ fueron $1 / 6 / 3 / 0.3$.

Las condiciones de síntesis empleadas para la obtención del material híbrido orgánico-inorgánico fueron las siguientes. Se prepararon dos disoluciones: la primera con el volumen total de TEOS, PDMS y la mitad del volumen de i-PrOH, y la segunda con la otra mitad del volumen de i- $\mathrm{PrOH}$, el $\mathrm{H}_{2} \mathrm{O}$ y el $\mathrm{HCl}$. Tras la homogeneización, las disoluciones se añadieron a un matraz a $80^{\circ} \mathrm{C}$. Transcurridos 60 minutos, la disolución se vertió y se cerró en un contenedor de plástico hasta su gelificación. Los exudados se retiraron y posteriormente el gel húmedo se secó a 50 y $120^{\circ} \mathrm{C}$ hasta observar peso constante. Las piezas del material híbrido resultante fueron blancas y monolíticas.

Estas piezas poseían tamaños de $100 \mathrm{~mm}$ x $50 \mathrm{~mm}$ x 5 $\mathrm{mm}$ y fueron utilizadas con su mismo tamaño o bien fueron trituradas con una granceadora a tamaños próximos de 1 $\mathrm{mm} \times 1 \mathrm{~mm} \times 1 \mathrm{~mm}$ o bien molidas en mortero de ágata o en atrición. En este último caso se emplearon bolas de zircona y como medio de molienda etanol. El tiempo de molienda empleado fue de dos horas y, transcurrido este tiempo, las bolas se retiran, se filtra y los polvos se secan en una estufa a $50{ }^{\circ} \mathrm{C}$. Finalmente, a fin de evitar aglomerados estos polvos se tamizan por debajo de $100 \mu \mathrm{m}$.

Independientemente del tamaño del material híbrido, éstos se introducen en un horno tubular (alúmina 99.99 $\%)$ de atmósfera controlada de nitrógeno $\left(50 \mathrm{~cm}^{3} \mathrm{~min}^{-1}\right)$, hasta la temperatura de $1100{ }^{\circ} \mathrm{C}$ durante 2 horas, utilizando velocidades de calentamiento y enfriamiento de $2^{\circ} \mathrm{Cmin}^{-1}$.

\subsection{Caracterización de los materiales}

Los vidrios de oxicarburo obtenidos fueron caracterizados desde un punto de vista estructural y microestructural. El análisis estructural fue llevado a cabo mediante las espectroscopías Infrarroja (FT-IR) y Raman, y difracción de rayos-X (DRX). Se determinó también el contenido en carbono, silicio y oxígeno. El análisis microestructural de los materiales se llevó a cabo mediante intrusión de mercurio, isotermas de adsorción-desorción de nitrógeno, microscopia electrónica de barrido (MEB) y densidad real.

Los espectros infrarrojos fueron obtenidos en un espectrómetro con transformada de Fourier (Perkin-Elmer modelo 1760x) utilizando la técnica de dilución en $\mathrm{KBr}$. En todos los casos se realizaron 10 barridos en el intervalo 4000-400 $\mathrm{cm}^{-1}$ con una resolución de $1 \mathrm{~cm}^{-1}$. Los espectros Raman se realizaron con un espectrómetro Raman (Renishaw 
modelo inVia) empleando como fuente de radiación un láser de ión $\operatorname{Ar}(\lambda=514 \mathrm{~nm})$. El equipo emplea como sistema de calibración silicio metálico que presenta una banda a 520 $\mathrm{cm}^{-1}$. Las muestras se enfocan empleando un microscopio óptico Leica (con el objetivo de 50 x) siendo el área de análisis aproximadamente de $1 \mu \mathrm{m}^{2}$. En todos los casos se llevan a cabo 10 barridos entre 200 y $3200 \mathrm{~cm}^{-1}$ con un tiempo de exposición de 20 s. Los DRX se obtuvieron con un difractómetro (Siemens, D5000) con un voltaje de $40 \mathrm{kV}$ y una intensidad de $20 \mathrm{~mA}$. El equipo emplea como fuente de radiación la línea $\mathrm{k}_{\alpha}$ del $\mathrm{Cu}(\lambda$ $=1.54060 \AA$ A). El programa de barrido fue registrado entre $10 \mathrm{y}$ $90^{\circ}(2 \Theta)$ en incrementos de $0.02^{\circ}$ y un tiempo de acumulación de $1 \mathrm{~s}$. El contenido en carbono fue determinado mediante un equipo analizador de carbono de la marca LECO, modelo CS-200. Tras añadir un acelerador, la muestra se combustiona a $2300{ }^{\circ} \mathrm{C}$ de manera que el carbono se transforma en $\mathrm{CO}_{2}$ y es cuantificado empleando una célula infrarroja. El contenido en silicio fue determinado mediante gravimetría de $\mathrm{SiO}_{2}$. La muestra primero se calcina y a continuación el residuo se funde empleando carbonato sódico y se le añade una disolución de $\mathrm{HCl}$ de manera que todos los componentes excepto la sílice se disuelven, finalmente el residuo sólido se calcina y se estima la sílice. El contenido en oxígeno de las muestras ha sido estimado por diferencia. El equipo empleado para la intrusión de mercurio fue el Autopore II 9215 (Micromeritics). Las isotermas de adsorción-desorción de nitrógeno se obtuvieron en un equipo Tristar 3000 (Micromeritics). La superficie de factura de los vidrios de oxicarburo de silicio se ha estudiado mediante MEB empleando un equipo Zeiss DSM 950. Las densidades reales se determinaron en un picnómetro de Helio tipo Monosorb Multipycnometer (Quantachrome Corporation). En las muestras obtenidas mediante molienda en ágata o en molino de atrición se ha determinado el tamaño de partícula mediante difracción láser utilizando un equipo Mastersizer S (Malvern), mezclando las muestras en etanol y posteriormente dispersándolas en ultrasonidos durante 5 minutos.

\section{RESULTADOS}

En este trabajo se ha evaluado cómo influye el tamaño del material híbrido (monolito o polvo) en las características del material de oxicarburo de silico final. Para ello se han analizado los vidrios de oxicarburo de silicio obtenidos mediante pirólisis a $1100{ }^{\circ} \mathrm{C}$ en atmósfera de nitrógeno. A continuación se presentan los resultados obtenidos tras llevar a cabo un estudio estructural y microestructural de dichos vidrios de oxicarburo de silicio.

\subsection{Microscopía electrónica de barrido}

En la Figura 1 se muestran las microfotografías obtenidas mediante MEB para todos los oxicarburos de silicio estudiados en este trabajo. El oxicarburo de silicio obtenido a partir del monolito es fundamentalmente macroporoso, con un tamaño de poro aproximado de $10 \mu \mathrm{m}$, presenta una morfología típica de agregado de partículas esféricas, dando como resultado grandes cavidades que interconectan unas zonas con otras. Esta microestructura se mantiene para el oxicarburo de silicio triturado en partículas de $1 \mathrm{~mm}$, así como para el molido en mortero de ágata, si bien, en éste último caso se pueden observar partículas esféricas fracturadas por efecto de la molienda. En todos estos casos se aprecian los macroporos existentes entre las partículas esféricas. Finalmente, como consecuencia de la molienda llevada a cabo en molino de atrición, el oxicarburo obtenido ha perdido los macroporos y se aprecia que ahora está constituido por partículas de geometría irregular, pero con un tamaño de partícula bastante homogéneo que se sitúa en torno a $3 \mu \mathrm{m}$.

\subsection{Análisis químico}

Los resultados del contenido en carbono y carbono libre, análisis químico, fórmula SiOC y tamaño del material para los oxicarburos de silicio del sistema TEOS/PDMS obtenidos a $1100{ }^{\circ} \mathrm{C}$ están recogidos en la TABLA 1 . Se ha empleado la Fórmula 1 para calcular la fórmula del oxicarburo de silicio a partir de la cual se puede estimar el carbono introducido en la red vítrea $\left(\mathrm{C}_{\text {oxi }}\right)$ y el que se encuentra como carbono libre $\left(\mathrm{C}_{\text {libre }}\right)$.

La muestra que ha sido procesada en forma de monolito es la que presenta el mayor porcentaje de carbono, pero cuando se lleva a cabo el triturado o la molienda en mortero de ágata se aprecia un gran descenso del contenido en carbono. Sorprendentemente, para la muestra que ha sido molida en molino de atrición el contenido en carbono vuelve a aumentar y es comparable al obtenido para el monolito.
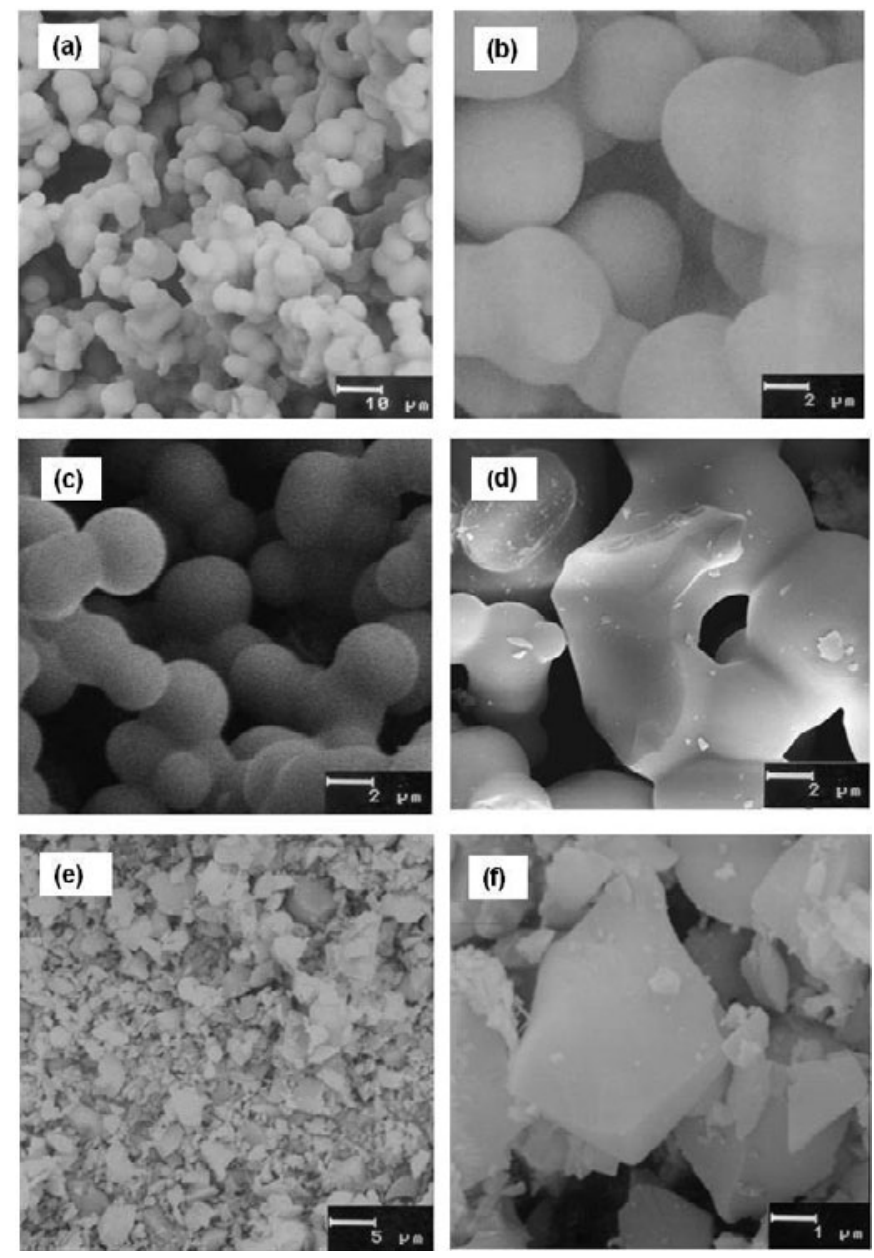

Figura 1: Micrografías de los oxicarburos de silicio obtenidos a partir de híbridos de diferente tamaño. a,b) Monolito 100x50x5 mm, c) triturado a 1x1x1 mm, d) molido con mortero de ágata $(16 \mathrm{~mm}), \mathrm{e}, \mathrm{f})$ molido por atrición $(3 \mathrm{~mm})$. 
TABla 1: TAMAÑo, \% CARBONO, ANÁLISIS QUíMICO, FÓRMULA DEL SIOC Y \% EN CARBONO LIBRE PARA LOS SIOC DEL SISTEMA TEOS/ PDMS OBTENIDOS A 1100 C.

\begin{tabular}{|c|c|c|c|c|c|c|}
\hline Tipo de procesamiento & Tamaño & $\mathbf{C}(\%) \pm \mathbf{0 . 0 6}$ & $\mathbf{S i}(\boldsymbol{\%}) \pm \mathbf{0 . 2}$ & $\mathbf{O}(\boldsymbol{\%}) \pm \mathbf{0 . 2}$ & Fórmula SiOC & $\mathbf{C}$ libre (\%) \\
\hline Monolito & $100 \mathrm{~mm}$ & 9.86 & 46.2 & 43.9 & $\mathrm{SiC}_{0.17} \mathrm{O}_{1.67}+0.33 \mathrm{C}$ & 66 \\
\hline Triturado & $1 \mathrm{~mm}$ & 8.54 & 46.4 & 45.0 & $\mathrm{SiC}_{0.15} \mathrm{O}_{1.70}+0.28 \mathrm{C}$ & 66 \\
\hline M. Ágata & $16 \mu \mathrm{m}$ & 8.25 & 45.6 & 46.2 & $\mathrm{SiC}_{0.11} \mathrm{O}_{1.77}+0.31 \mathrm{C}$ & 74 \\
\hline M. Atrición & $3 \mu \mathrm{m}$ & 9.50 & 46.1 & 44.4 & $\mathrm{SiC}_{0.15} \mathrm{O}_{1.69}+0.33 \mathrm{C}$ & 68 \\
\hline
\end{tabular}

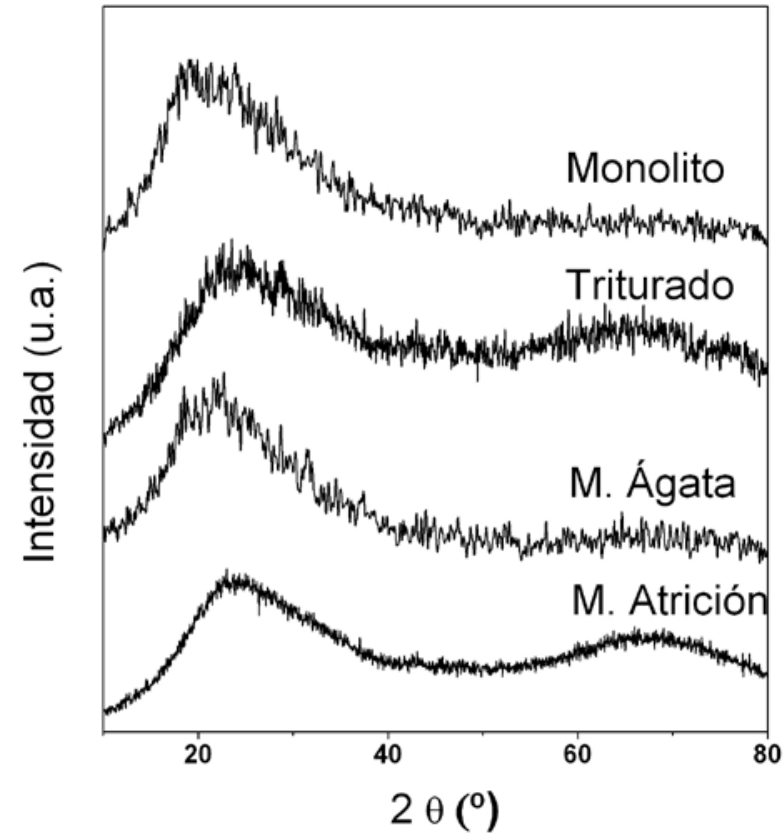

Figura 2: Difractogramas de RX para los oxicarburos de silicio del sistema TEOS/PDMS obtenidos a $1100^{\circ} \mathrm{C}$.

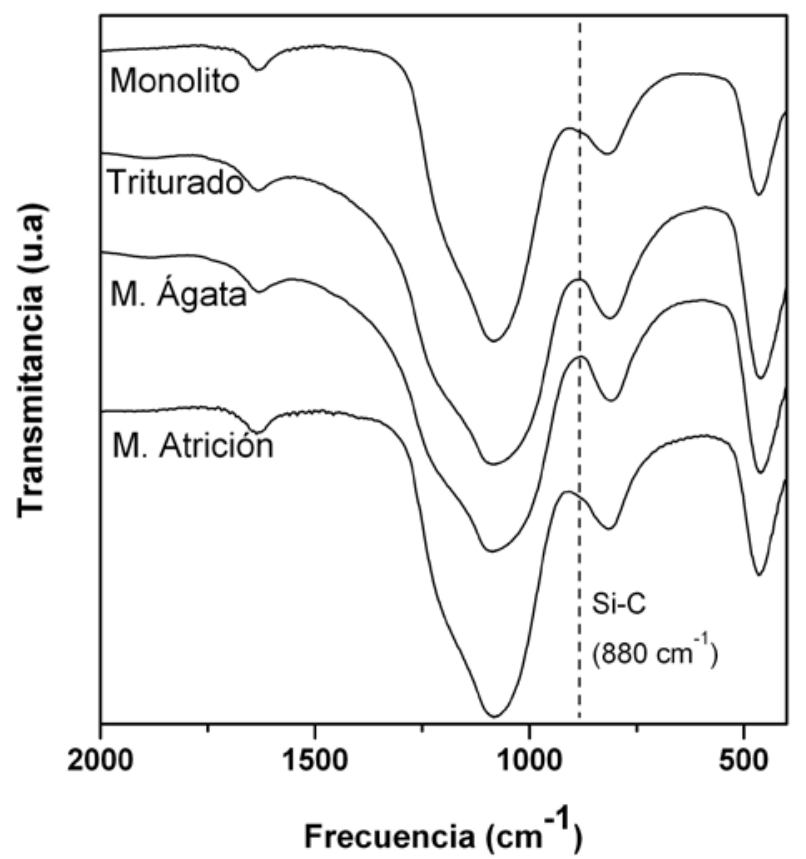

Figura 3: Espectros de FT-IR para los oxicarburos de silicio del sistema TEOS/PDMS, obtenidos a $1100{ }^{\circ} \mathrm{C}$.
Cuando se rompe el monolito, ya sea para el caso de la muestra cortada o molida en mortero de ágata, aumenta la superficie reactiva del material. Esto hace que durante la pirólisis se produzca en mayor medida la despolimerización del PDMS, lo que provoca una mayor pérdida de carbono y por lo tanto una menor incorporación de carbono en la red vítrea. Sin embargo, cuando se ha llevado a cabo la molienda en atrición alcanzando un tamaño de partícula de hasta $3 \mu \mathrm{m}$ se produce una gran homogeneización en la muestra que da lugar a que durante el proceso de pirólisis la pérdida de PDMS sea únicamente comparable a la que experimenta el monolito. El hecho de que la molienda en atrición se lleve a cabo en etanol puede influir en cierto sentido para que la pérdida de PDMS sea únicamente comparable a la que se produce en el monolito. El aumento o disminución de carbono total se traduce en un aumento o disminución de carbono incorporado en la red vítrea o embebido dentro de ella en forma de carbono libre.

\subsection{Difracción de rayos $X$}

Los DRX para los oxicarburos de silicio obtenidos a $1100^{\circ} \mathrm{C}$ están representados en la Figura 2. Para todos los casos dichos difractogramas son muy similares presentando una banda centrada a $22^{\circ}$, característica de materiales vítreos basados en sílice. Si bien, esta banda presenta una asimetría hacia ángulos mayores, resultado que debe ser asociado a la fase de carbono libre tipo grafito $(27)(2 \Theta=26.5,44.6$ y 54.7$)$. No se aprecia la presencia de picos asociados a especies de carburo de silicio cristalino (28) ni de $\beta-\operatorname{SiC}\left(2 \Theta=35,60\right.$ y $\left.75^{\circ}\right)$.

\subsection{Espectroscopías Infrarroja y Raman}

En la Figura 3 están representados los espectros de FT-IR de los oxicarburos de silicio obtenidos a $1100{ }^{\circ} \mathrm{C}$. Todos ellos presentan las bandas típicas de vidrio de oxicarburo de silicio (2) observándose una banda ancha e intensa que corresponde a la tensión asimétrica del enlace Si-O-Si con sus dos componentes longitudinal y transversal, a 1170 y 1080 $\mathrm{cm}^{-1}$ ), respectivamente. Se observa también la vibración de tensión simétrica del tetraedro $\mathrm{SiX}_{4}(\mathrm{X}=\mathrm{C}, \mathrm{O})$ situada a 810 $\mathrm{cm}^{-1}$ y la de deformación del enlace O-Si-O, centrada en 460 $\mathrm{cm}^{-1}$. Cabe destacar que únicamente en los SiOC obtenidos como monolito o con molino de atrición se puede intuir ligeramente un hombro a $880 \mathrm{~cm}^{-1}$, que está asociado a la vibración del enlace $\mathrm{Si}-\mathrm{C}$ en $\beta$-SiC (29). Este resultado es indicativo de una mayor incorporación de carbono en la red mixta del oxicarburo de silicio, tal y como se ha observado en los resultados de análisis químico de las muestra (TABLA 1). 
En la Figura 4 se presentan los espectros Raman para los oxicarburos de silicio obtenidos a $1100{ }^{\circ} \mathrm{C}$. En todos ellos se observan dos bandas situadas aproximadamente a 1360 y $1580 \mathrm{~cm}^{-1}$, denominadas bandas $D$ y $G$, características del carbono tipo grafito (30) y atribuidas a la fase de carbono libre, embebida dentro de la matriz del oxicarburo de silicio.

De acuerdo con Knight y White (31), a partir de la relación de intensidades $I(D) / I(G)$ se puede calcular el tamaño de los dominios de grafito (Ecuación I). Hay que tener en cuenta que esta expresión es válida únicamente para una radiación láser de $\lambda=514 \mathrm{~nm}$ como es la empleada en este trabajo. Por otro lado, la anchura de las bandas es inversamente proporcional al grado de ordenación (31).

$$
L(n m)=4.4(\lambda) x\left[\frac{I(D)}{I(G)}\right]^{-1}
$$

\section{Ecuación I}

Para materiales tipo grafito con un elevado grado de desorden, la banda $G$ es muy ancha y su posición se desplaza hasta $\sim 1600 \mathrm{~cm}^{-1}$. Este hecho ha sido explicado por la presencia de una banda adicional la denominada banda $\mathrm{D}^{\prime}\left(1620 \mathrm{~cm}^{-1}\right)$ que también se asocia al desorden (32). Por lo tanto, para este tipo de materiales esta ancha banda es contribución de la banda G y la banda D'. El desdoblamiento de esta banda en sus dos componentes se produce al aumentar el orden de los materiales o al disminuir la longitud de onda de la radiación láser incidente (33).

Los espectros Raman de la Figura 4 muestran picos muy anchos lo que indica que la fase de carbono libre de estos oxicarburos de silicio corresponde con carbono tipo grafito altamente desordenado. A partir de la Ecuación I se ha calculado el tamaño de los dominios (TABLA 2), estando alrededor de $3.5 \mathrm{~nm}$ para todos los materiales. En la TABLA 2 también se han incluido los desplazamientos y las anchuras de las bandas D y G, respectivamente. Todos estos valores confirman que la fase de carbono libre es muy similar independientemente del tamaño del híbrido precursor.

No obstante, se puede observar en estos resultados que, a medida que se disminuye el tamaño de partícula la banda $G$ presenta un menor desplazamiento, relativo al que poseería el grafito altamente ordenado a $1580 \mathrm{~cm}^{-1}$, (la variación se produce desde 1586 hasta $1573 \mathrm{~cm}^{-1}$ ), lo que estaría indicando una mayor ordenación. Este resultado se correlaciona con un ligero aumento en el tamaño de los dominios de carbono tipo grafito desde 3.44 hasta $3.66 \mathrm{~nm}$ al pasar de monolito a molido en atrición.

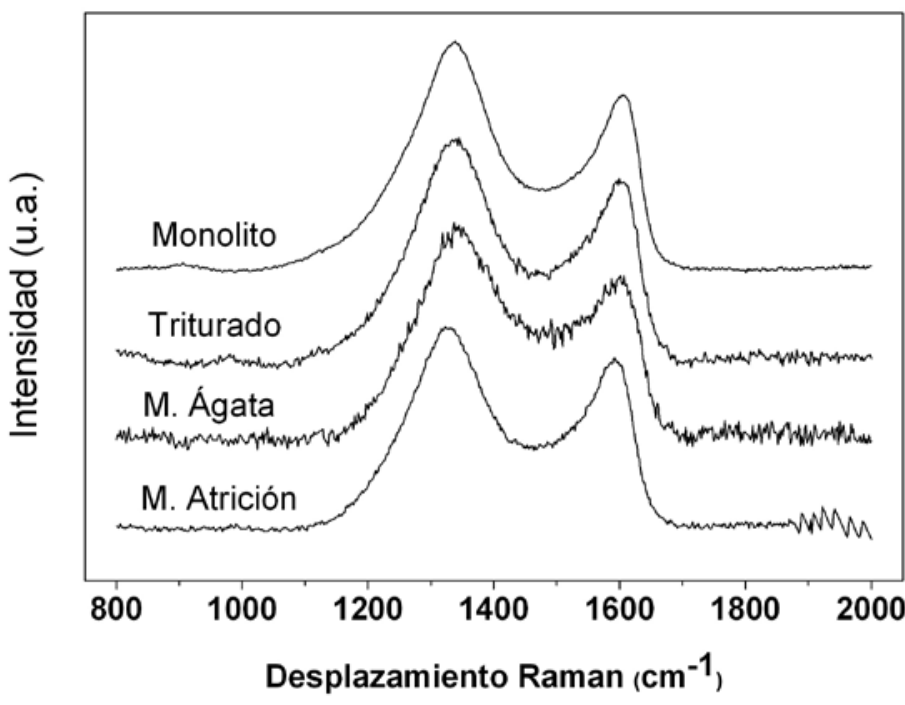

Figura 4: Espectros Raman para los oxicarburos de silicio del sistema TEOS/PDMS, obtenidos a $1100^{\circ} \mathrm{C}$.

\subsection{Porosimetría de Mercurio, Adsorción de Nitrógeno y Picnometría de Helio}

Los resultados obtenidos de la caracterización microestructural (Porosimetría de mercurio, adsorcióndesorción de nitrógeno y picnometría de helio) llevada a cabo, están recogidos en la TABLA 3.

De forma general, la porosimetría de mercurio se rige por la ecuación de Washburn (34) (Ecuación II):

$$
r=-\frac{2 \gamma \cos \theta}{P}
$$

\section{Ecuación II}

Donde $r$ es el radio de los poros cilíndricos, $P$ es la presión a la cual el mercurio penetra en los poros, $\gamma$ es la tensión superficial del mercurio $(0.485 \mathrm{~N} / \mathrm{m})$ y $\theta$ es el ángulo de contacto entre el menisco que forma el mercurio y una superficie plana y no metálica $\left(\theta \approx 140^{\circ}\right)$.

Los materiales estudiados en este trabajo presentan morfologías muy distintas, desde piezas monolíticas de gran tamaño a polvos obtenidos por molienda, por lo que debe tenerse en cuenta que la intrusión de mercurio es el resultado de la intrusión tanto dentro de los poros como en el espacio

\begin{tabular}{|c|c|c|c|c|c|c|}
\hline Tipo de procesamiento & Banda D $\left(\mathrm{cm}^{-1}\right)$ & Anchura D $\left(\mathrm{cm}^{-1}\right)$ & Banda G $\left(\mathrm{cm}^{-1}\right)$ & Anchura G $\left(\mathrm{cm}^{-1}\right)$ & $\mathrm{I}(\mathrm{D}) / \mathrm{I}(\mathrm{G})$ & $\mathrm{L}(\mathrm{nm})$ \\
\hline Monolito & 1338 & 150 & 1586 & 90 & 1.28 & 3.44 \\
\hline Triturado & 1340 & 157 & 1581 & 100 & 1.24 & 3.56 \\
\hline M. Ágata & 1351 & 161 & 1577 & 110 & 1.33 & 3.30 \\
\hline M. Atrición & 1332 & 156 & 1573 & 100 & 1.20 & 3.66 \\
\hline
\end{tabular}

TABla 2: Resultados RAMAN PARA LOS SIOC DEL SISTEMA TEOS/PDMS, OBTENIDOS A $1100{ }^{\circ} \mathrm{C}$. 
entre partículas. Tal y como establecen Pirard et al. $(35,36)$, en ciertos materiales (aerogeles, xerogeles o negro de carbono), al utilizar la técnica de porosimetría de mercurio a bajas presiones, dichos materiales experimentan una compactación o colapso que hace que la ecuación de Washburn no pueda ser aplicada ya que da lugar a una sobreestimación del diámetro de poro. A partir de una presión de transición $\left(\mathrm{P}_{\mathrm{t}}\right)$, característica de la microestructura de cada material, el mercurio puede penetrar dentro de los poros pequeños que no han sido destruidos durante la compresión a bajas presiones. Este hecho puede ser observado en la curva de presiones mediante un cambio en la pendiente de dicha curva para una presión de transición dada $\left(P_{t}\right)$. Conocido el valor de esta presión de transición, se puede calcular la constante $\left(K_{f}\right)$ mediante la Ecuación III.

$$
K_{f}=\frac{4 \gamma \cos \theta}{P_{t}^{0.75}}
$$

\section{Ecuación III}

Para la presión de transición se cumplen tanto la ecuación de Washburn (Ecuación II) como la ecuación de densificación (Ecuación III). Por lo tanto, es fácil la determinación de $K_{f}$ en muestras que experimentan este comportamiento mixto de densificación e intrusión y, a su vez, permite calcular la distribución de tamaño $(L)$ durante la etapa de densificación.

$$
L=\frac{K_{f}}{P^{0.25}}
$$

\section{Ecuación IV}

Este tipo de corrección de la ecuación de Washburn ha sido empleado para las muestras en polvo de este trabajo (37), habiéndose calculado la sobreestimación que se produce al considerar la compactación de las partículas. Mediante la corrección propuesta $(35,36)$, se determinan dos regímenes diferentes: en primer lugar (para presiones menores) se produce una compactación de las partículas o colapso de la estructura y, en segundo lugar, se da paso a la intrusión de mercurio dentro de los huecos o poros del material. La primera etapa está regida por lo que se denomina ecuación de densificación y, tras alcanzarse una presión de transición $\left(P_{t}\right)$, tiene validez la ecuación de Washburn en la segunda etapa.

En la Figura 5 se muestran las distribuciones de tamaño de poro obtenidas mediante intrusión de mercurio. La muestra monolítica presenta una distribución monomodal con un

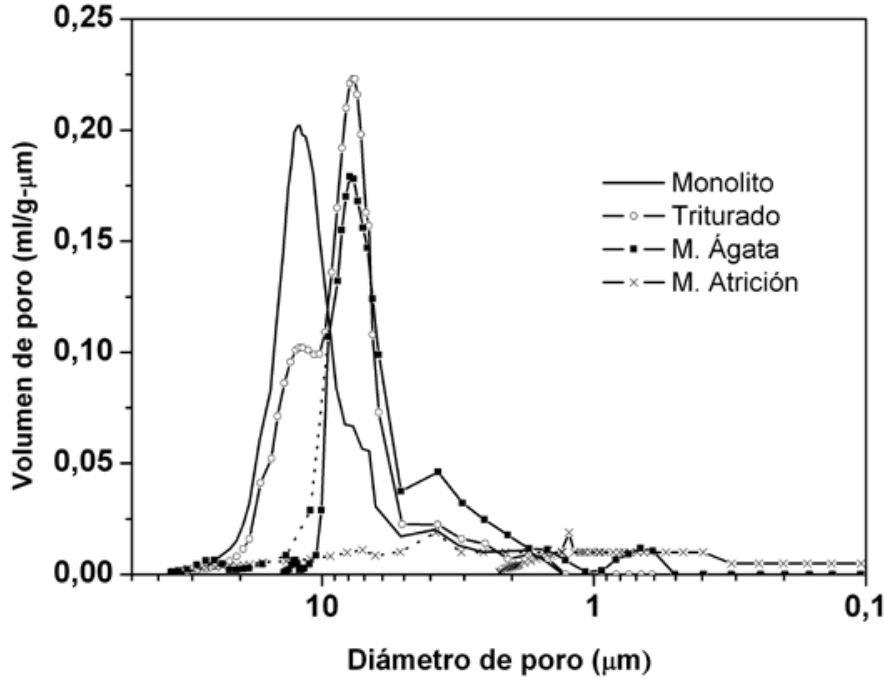

Figura 5: Distribuciones de tamaño de poro para los oxicarburos de silicio del sistema TEOS/PDMS.

diámetro de poro en torno a las $12 \mu \mathrm{m}$. Cuando se observan las muestras que han sido trituradas/molturadas se produce una disminución de dicho tamaño. Esto, lógicamente, también se traduce en una disminución de la porosidad total (TABLA 3). La muestra que ha sido triturada presenta una distribución de poros bimodal con centros a 12 y $8 \mu \mathrm{m}$. Posteriormente la muestra que ha sido molida en un mortero de ágata vuelve a ser monomodal con poros centrados a $8 \mu \mathrm{m}$. Por último, la muestra molida con molino de atrición prácticamente no presenta porosidad como se ha podido observan mediante microscopía electrónica (Figura 1(e,f)).

Las isotermas obtenidas mediante adsorción-desorción de nitrógeno (no se muestran en este trabajo) han sido todas de tipo II, correspondientes a materiales que no presentan ni mesoporos ni microporos. En general, los valores de área BET observados son muy bajos excepto para los oxicarburos de silicio obtenidos a partir de los híbridos molidos por atrición, donde el área BET aumenta hasta $8 \mathrm{~m}^{2} \mathrm{~g}^{-1}$ : (TABLA 3).

La picnometría de helio da unos resultados muy similares de densidad real para todos los vidrios de oxicarburo obtenidos, estando próximos a $2.32 \mathrm{gcm}^{-}{ }^{3}$. Esto está indicando

TABLA 3: RESULTADOS MICROESTRUCTURALES PARA LOS SIOC OBTENIDOS A PARTIR DE HÍBRIDOS DEL SISTEMA TEOS/ PDMS PIROLIZADOS A $1100{ }^{\circ} \mathrm{C}$.

\begin{tabular}{|c|c|c|c|c|c|}
\hline Técnica & Propiedad & Monolito & Triturado & M. Ágata & M. Atrición \\
\hline \multicolumn{2}{|c|}{ Tamaño } & $100 \times 50 \times 5(\mathrm{~mm})$ & $1 \times 1 \times 1(\mathrm{~mm})$ & $16(\mu \mathrm{m})$ & $3(\mu \mathrm{m})$ \\
\hline \multirow{2}{*}{ Ads/Des.N $\mathbf{N}_{2}$} & Superficie BET $\left(\mathrm{m}^{2} \mathrm{~g}^{-1}\right)$ & 1.35 & 0.83 & 0.47 & 8.01 \\
\hline \multirow{2}{*}{ Porosimetria Hg } & Porosidad $(\%)$ & 77 & 43 & 46 & $\sim 0$ \\
\cline { 2 - 6 } & Diámetro de poro $(\mu \mathrm{m})$ & 11.8 & 9.40 & 7.67 & - \\
\hline \multirow{2}{*}{ Picnometría de He } & Densidad real $\left(\mathrm{gml}^{-1}\right)$ & 2.33 & 2.30 & 2.32 & 2.32 \\
\hline
\end{tabular}


que aparte del diferente tamaño del híbrido (monolito o polvo), el proceso de pirólisis ha producido la transformación orgánico-inorgánica del material de manera satisfactoria y que es independiente de la microestructura o del tamaño del material de partida (TABLA 3).

\section{DISCUSIÓN}

Aunque los resultados obtenidos por DRX indican que todos los materiales presentan estructura amorfa o vítrea independientemente del tamaño del material híbrido utilizado, sin embargo si se puede concluir que dicho tamaño influye en las características de los materiales de SiOC obtenidos tras el proceso de pirólisis.

En primer lugar, puede apreciarse que el material que se ha pirolizado en monolito es el que presenta un mayor porcentaje de carbono incorporado en la red vítrea, tal y como puede concluirse a partir tanto de los resultados obtenidos según la fórmula del oxicarburo, como a partir de los espectros infrarrojos (hombro situado a $880 \mathrm{~cm}^{-1}$ ). Cuando el material se tritura o bien se muele en mortero de ágata la superficie aumenta y una gran parte de PDMS se pierde durante las reacciones de despolimerización, lo que hace que disminuya el contenido en carbono incorporado en la red. Sin embargo, cuando el híbrido se muele mediante atrición se obtiene una incorporación de carbono en red similar a la del monolito. El pequeño tamaño de partícula conseguido mediante atrición, 3 $\mu \mathrm{m}$, la gran homogeneidad de los polvos y el medio empleado en la molienda (etanol) parece que están contrarrestando la pérdida de carbono debida a la despolimerización del PDMS. El etanol se puede haber quedado adsorbido sobre las partículas del híbrido de manera que durante la pirolisis se reduce y evita que el PDMS se pierda. De hecho los valores conseguidos son similares a los que se han obtenido mediante la pirolisis de la pieza en monolito.

En estudios anteriores Turquat et al. (38) llevaron a cabo la síntesis de SiOC a partir de polvos de silicio cristalino, sílice y grafito empleando un molino de bolas. Para unas determinadas condiciones de molienda y tamaños de partícula inferiores a $43 \mathrm{~mm}$ de los materiales de partida, se observó que como consecuencia del proceso mecánico se producía inicialmente la amorfización de las materias primas seguido de la formación de enlaces Si-C y finalmente la oxidación de dichos enlaces para dar lugar al vidrio de oxicarburo de silicio. En este sentido algo similar podría estar ocurriendo para los híbridos orgánico-inorgánicos del sistema TEOS/PDMS, según sean molidos en mortero de ágata o por atrición. En el primer caso, la disminución del tamaño de partícula únicamente da lugar a una disminución del contenido de carbono incorporado en la red como consecuencia de un aumento de la superficie de la partícula. Sin embargo, cuando la molienda se ha llevado a cabo en mayor extensión, la disminución del tamaño de partícula hasta $3 \mu \mathrm{m}$, la gran homogeneidad conseguida y la posible adsorción de etanol en las partículas da lugar a una disminución de la pérdida de carbono favoreciendo la formación de vidrio de oxicarburo de silicio durante la pirolisis. Para este caso, incluso se ha observado un aumento en el tamaño de los dominios de grafito y en el grado de ordenación de los mismos, tal y como se observa por la disminución de la posición de la banda $G$ y del tamaño del dominio del cristal de grafito, recogidos en la TABLA 2.
Como era de esperar, las características microestructurales también varían en función del tamaño del material híbrido de partida. Así, se observa que los macroporos (12 $\mu \mathrm{m})$ presentes en la muestra obtenida como monolito desaparecen a medida que el material se va triturando o moliendo, pasando a $8 \mu \mathrm{m}$ y llegando prácticamente a desaparecer cuando se muele por atrición. Además, la muestra molida mediante atrición ha perdido la estructura de agregado de partículas interconectadas $y$, ahora está formada únicamente por partículas de $3 \mu \mathrm{m}$. Por lo tanto, se puede concluir que para obtener un material de SiOC sin poros y con el mayor contenido posible de carbono en red, conviene llevar a cabo la molienda en atrición.

\section{CONCLUSIONES}

La caracterización estructural y microestructural llevada a cabo en este trabajo, ha servido para evaluar la repercusión que tiene el tamaño del híbrido orgánico-inorgánico del sistema TEOS/PDMS durante el proceso de pirólisis. Así, a medida que el monolito se tritura o se muele, aumenta la superficie del material y se favorecen las reacciones de despolimerización del PDMS, lo que se traduce en una menor incorporación del carbono en la red vítrea. Sin embargo, cuando la molienda se lleva a cabo en atrición, se ha conseguido mayor homogeneidad en las partículas, que hace que la pérdida de carbono sea menor. Este resultado se concluye mediante análisis químico (fórmula estequiométrica de oxicarburo de silicio) y mediante FT-IR (hombro situado a $880 \mathrm{~cm}^{-1}$ y asignado a la formación de enlaces $\mathrm{Si}-\mathrm{C}$ en el $\beta$-SiC). Mediante Raman, se concluye que la fase de carbono libre corresponde en todos los casos con carbono tipo grafito altamente desordenado, presentando unos dominios de $\approx 3.5 \mathrm{~nm}$. En los materiales obtenidos mediante atrición se aprecia un ligero aumento del orden así como un mayor tamaño de los dominios de grafito.

El oxicarburo de silicio obtenido en monolito presenta una morfología típica de agregado de partículas esferoidales con poros centrados en $12 \mu \mathrm{m}, \mathrm{y}$ a medida que se muele disminuye hasta $8 \mu \mathrm{m}$, sin perder la forma esférica de las partículas. Finalmente, cuando se muele en atrición se eliminan completamente los poros y la forma de las partículas es irregular con un tamaño de $3 \mu \mathrm{m}$.

\section{AGRADECIMIENTOS}

Este trabajo ha sido realizado dentro de los proyectos de investigación financiados por el Ministerio de Educación y Ciencia de España de referencia CTQ2006-15692-C02-02 y MAT2009-14450.

\section{BIBLIOGRAFIA}

(1) C.G. Pantano, A.K. Sinhg, H.Zhang, "Silicon oxycarbide glasses," J. Sol-Gel Sci. Tech., 14, 7-25 (1999).

(2) G.M. Renlund, S. Prochazka, F.H. Doremus, "Silicon oxycarbide glasses: part II. Structure and properties," J. Mater. Res., 6[12], 2723-34 (1991).

(3) G.D. Sorarú, E. Dallapiccola, G. D'Andrea, "Mechanical characterization of sol-gel-derived silicon oxycarbide glasses," J. Am. Ceram. Soc., 79, 2074-79 (1996). 
(4) N. Suyal, T. Krajewski, M. Menning, "Sol-gel synthesis and microstructural characterization of silicon oxycarbide glass sheets with high fracture strength and high modulus," J. Sol-Gel Sci. Tech., 13, 995-999 (1998).

(5) S. Walter, G.D. Soraru, H. Bréquel, S. Enzo, "Microstructural and mechanical characterization of sol gel-derived Si-O-C," J. Eur. Ceram. Soc., 22, 2389-2400 (2002).

(6) C. Moysan, R. Riedel, R. Harshe, T. Rouxel, F. Augereau, " Mechanical characterization of a polysiloxane-derived SiOC glass," J. Eur. Ceram. Soc., 27, 397-403 (2007).

(7) G.D. Sorarù, D. Suttor, “High temperature stability of sol-gel derived SiOC glasses," J. Sol-Gel. Sci. Tech., 14, 69-74 (1999).

(8) T. Rouxel, G. Massouras, G.D. Sorarù, "High temperature behavior of a gelderived SiOC glass: Elasticity and viscosity," J. Sol-Gel. Sci. Tech., 14, 87-94 (1999).

(9) G.M. Renlund, S. Prochazka, F.H. Doremus, "Silicon oxycarbide glasses: part I. Preparation and chemistry," J. Mater. Res., 6[12], 2716-22 (1991).

(10) C.M. Brewer, D.R. Bujalski, V.E. Parent, K. Su, G.A. Zank, "Insights into the oxidation chemistry of SiOC ceramic derived from silsesquioxanes," $J$. Sol-Gel. Sci. Tech., 14, 49-68 (1999).

(11) G. Chollon, "Oxidation behaviour of ceramic fibres from the Si-C-N-O system and related sub-systems," J. Eur. Ceram. Soc., 20, 1959-74 (2000).

(12) S. Modena, G.D. Soraru, Y. Blue, R. Raj, "Passive oxidation of an effluent system: The case of polymer-derived SiCO," J. Am. Ceram. Soc., 88 [2], 339345 (2005).

(13) A.M. Wilson, G. Zank, K. Eguchi, W. Xing, B. Yates, J. R. Danh, "Pore creation in silicon oxycarbides by rinsing in dilute hydrofluoric acid," Chem. Mater., 9, 2139-2144 (1997)

(14) G.D. Sorarù, S. Modena, E. Guadagnino, P. Colombo, J. Egan, C. Pantano, "Chemical durability of silicon oxycarbide glasses," J. Am. Ceram. Soc., 85 [6], 1529-36 (2002).

(15) A. Scarmi, G.D. Soraru, R. Raj, "The role of carbon in unexpectec visco(an) elastic behavior of amophous silicon oxicarbide above $1273 \mathrm{~K}, "$ J. NonCryst. Solids, 351, 2238-2243 (2005).

(16) R. Riedel, L. Toma, E. Janssen, J. Nuffer, T. Melz, H. Hanselka, “Piezoresistive effect in SiOC ceramics for integrated pressure sensors," J. Am. Ceram. Soc., 93 [4], 920-924 (2010).

(17) R. Riedel, G. Mera, R. Hauser, A. Klonczynski, "Silicon-based polymerderived ceramics: synthesis properties and applications-A review," J. Ceram. Soc. Jpn., 114 [6], 425-444 (2006).

(18) G.D. Soraru, “Silicon Oxycarbide Glasses from Gels," J. Sol-Gel Sci. Tech., 2, 843-848 (1994).

(19) R.J.P. Corriu, D. Leclercq, P.H. Mutin, a. Vioux, ${ }^{{ }^{29} \mathrm{Si} i}$ nuclear magnetic resonance study of the structure of silicon oxycarbide glasses derived from organosilicon precursors," J. Mater. Sci., 30, 2313-18 (1995).

(20) H-J. Kleebe, Y.D. Blum, "SiOC ceramic with high excess free carbon," J. Eur. Ceram. Soc., 28, 1037-1042 (2008.)

(21) G.D. Sorarù, G. D'Andrea, R. Campostrini, F. Babonneua, G. Mariotto, "Structural characterization and high-temperature behavior of silicon oxycarbide glasses prepared from sol-gel precursors containing $\mathrm{Si}-\mathrm{H}$ bonds," J. Am. Ceram. Soc., 78[2], 379-87 (1995).
(22) P.H. Mutin "Control of the Composition and Structure of Silicon Oxycarbide and Oxynitride Glasses Derived from Polysiloxanes Precursors," J. Sol-Gel Sci. Tech., 14, 27-38 (1999).

(23) M.A. Mazo, “Nuevo Procedimiento de Obtención de vidrios de oxicarburo. Estudio de los mecanismos del proceso" Tesis doctoral . Madrid (2008).

(24) P. Colombo, T.E. Paulson, C.G. Pantano, "Atmosphere effects in the processing of silicon carbide and silicon oxycarbide thin films and coatings," J. Sol-Gel Sci. Tech., 2, 601-604 (1994).

(25) R. Peña-Alonso, L.Tellez, A. Tamayo, "Silicon-titanium oxycarbide glasses as bimodal porous inorganic membranes," J. Eur. Ceram. Soc., 27 [2-3], 397403 (2007).

(26) J. Parmentier, G.D. Soraru, F. Babonneau, "Influence of the microstructure on the high temperature behavior of gel-derived SiOC glasses," J. Eur. Ceram. Soc., 21, 817-824 (2001).

(27) M.A. Schiavon, S.U.A. Redondo, S.R.O. Pina, I.V.P. Yoshida, "Investigation on kinetics of thermal decomposition in polysiloxane networks used as precursors of silicon oxycarbide glasses," J. Non-Cryst. Solids., 304, 92-100 (2002).

(28) K.R. Carduner, S.S. Shinozaki, M.J. Rokosz, C.R. Peters and T.J. Whalen, "Characterization of $\beta$ - Silicon carbide by silicon-29 solid-state NMR, transmission electron microscopy, and powder X-ray diffraction," J. Am. Ceram. Soc., 73 [8], 2281-2286 (1990).

(29) I. Hasegawa, T. Nakamura, S. Motojima, M. Kajiwara, " Synthesis of silicon carbide fibers by sol-gel processing," J. Sol-Gel Sci. Tech., 8, 577-579 (1997).

(30) F. Tunistra, J.L. Koening, "Raman spectrum of graphite," J. Chem. Phys., 53 [3], 1126-1130 (1970).

(31) D.S. Knight, W.B. White, "Characterization of diamond films by Raman spectroscopy," J. Mater. Res., 4 [2], 385-393 (1989).

(32) R. Vidano, D.B. Fischbach, "New lines in the Raman spectra of carbons and graphite," J. Am. Ceram. Soc., 61 [1-2], 13-17 (1978).

(33) L.G. Cançado, K. Takai, T. Enoki, M. Endo, Y.A. Kim, H. Mizusaki, A. Jorio, L.N. Coelho, R. Magalhāes-Paniago, M.A. Pimenta, "General equation for the determination of the crystalline size $\mathrm{L}_{\mathrm{a}}$ of nanographite by Raman spectroscopy," Appl. Phys. lett., 88, 1631061-1631063 (2006).

(34) E.W. Washburn, "Note on a method of determining the distribution of pore sizes in a porous material," Proc. Nat. Acad. Sci., 7, 115-116 (1921).

(35) R. Pirard, S. Blacher, F. Brouers, J.-P. Pirard, "Interpretation of mercury porosimetry applied to aerogels," J. Mater. Res., 10 [8], 2114-2119 (1995).

(36) R. Pirard, B. Heinrichs, J.-P- Pirard, "Mercury porosimetry applied to low density xerogels" en McEnaney, T.J. Mays, J. Rouquerol, F. RodriguezReinoso, K.S.W. Sing, K.K. Unger (Eds.) Characterisation of porous solids IV, The Royal Society of Chemistry, Cambridge 460-466 (1997).

(37) R. Pirard, C. Alié, J.-P. Pirard, "Characterization of porous texture of hyperporous material by mercury porosimetry using densification equation," Powd. Tech., 128, 242-247 (2002).

(38) C. Turquat, H. Bréquel, H-J.Kleebe, F. Babonneau, S. Enzo, "Investigation of SiOC glasses synthetized with extensive ball milling," J. Non-Cryst. Solids, 319, 117-128 (2003).

Recibido: $15 / 11 / 2010$

Aceptado: 28/2/2012 